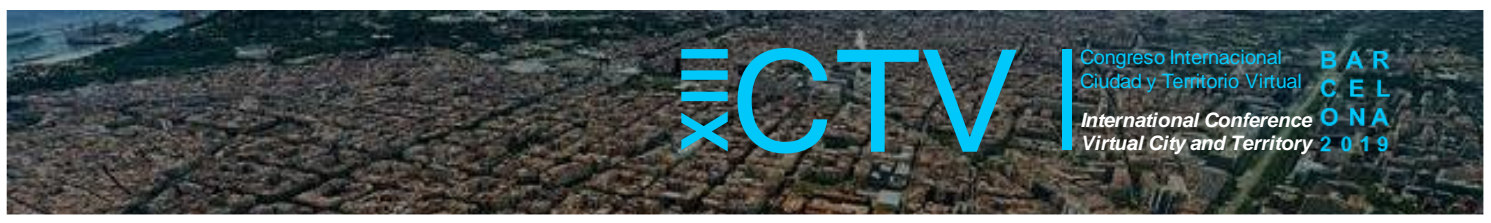

\title{
POBREZA ENERGÉTICA Y SEGREGACIÓN ESPACIAL: NUEVAS DIMENSIONES URBANAS PARA LA DESIGUALDAD
}

\author{
Encinas, Felipe ${ }^{1,2 *}$; Aguirre, Carlos ${ }^{3}$; Truffello, Ricardo ${ }^{2,4,5}$; Puig, Isidro ${ }^{4}$; Hidalgo, Rodrigo ${ }^{6}$
}

Remisión inicial: 2019-05-31; Remisión definitiva: 2019-10-13; Publicación: 2019-12-21

Citación: Encinas, F. et al. (2019). Pobreza energética y segregación espacial: nuevas dimensiones urbanas para la desigualdad. En XIII CTV 2019 Proceedings: XIII International Conference on Virtual City and Territory: "Challenges and paradigms of the contemporary city": UPC, Barcelona, October 2-4, 2019. Barcelona: CPSV, 2019, p. 8703. E-ISSN 2604-6512. DOI http://dx.doi.org/10.5821/ctv.8703

\section{Resumen}

El concepto de pobreza energética nace a principios de los años 90 en el Reino Unido como la incapacidad de obtener en un hogar la cantidad adecuada de servicios energéticos por el 10\% del ingreso familiar (Boardman 1991). Esta definición posteriormente ha sido complementada con aspectos constructivos y de habitabilidad, a partir de, por ejemplo, el establecimiento de una temperatura límite para la obtención de confort (DOE 1996).

En la literatura internacional más reciente es posible encontrar definiciones con un fuerte sesgo interdisciplinar a partir de la accesibilidad a 3 umbrales de vulnerabilidad: tecnológico, físico y económico (González-Eguino 2015). Este último es el más ampliamente utilizado en países desarrollados, principalmente asociado al consumo de calefacción para uso doméstico (concepto más preciso de "fuel poverty"), aunque se reconoce que tiende a subestimar la cantidad de hogares que efectivamente no puede costear el consumo energético para estas mínimas condiciones de habitabilidad, o la provisión de otras demandas de energía, que constituyen el denominado umbral tecnológico (Mould and Baker 2017; Bouzarovski and Petrova 2015). Por otra parte, el umbral físico ha sido estudiado de manera más reciente desde el confort térmico, específicamente en su relación con la vulnerabilidad y salud, encontrando correlaciones significativas con respecto a las características constructivas de las viviendas (Grey, Jiang, and Poortinga 2015; Atsalis et al. 2016).

En este contexto, la incorporación de metodologías de segregación socio-espacial para enriquecer este concepto es de muy reciente data y todavía no ha sido aplicado a nivel nacional. Este enfoque resulta importante, puesto que permite identificar zonas de aglomeración de pobreza energética, aspecto importante para el diseño de políticas urbanas.

\section{Abstract}

The concept of energy poverty was born in the early 1990s in the United Kingdom as the inability to obtain an adequate amount of energy services at home for $10 \%$ of family income (Boardman 1991).

This definition has subsequently been complemented with constructive and habitability aspects, for example, from the establishment of a limit temperature for obtaining comfort (DOE 1996). In the most recent international literature, it is possible to find definitions with a strong interdisciplinary bias based on accessibility to 3 vulnerability thresholds: technological, physical and economic (González-Eguino 2015). The latter is the most widely used in developed countries, mainly associated with the consumption of heating for domestic use (more precise concept of "fuel poverty"), although it is recognized that it tends to underestimate the number of households that cannot effectively afford energy consumption for these minimum habitability conditions, or the provision of other energy demands, which constitute the so-called technological threshold (Mold and Baker 2017; Bouzarovski and Petrova 2015). On the other hand, the physical threshold has been studied more recently from thermal comfort, specifically in its relationship with vulnerability and health, finding significant correlations with respect to the constructive characteristics of the houses (Gray, Jiang, and Poortinga 2015; Atsalis et al. 2016).

\footnotetext{
${ }^{1}$ Escuela de Arquitectura; Facultad de Arquitectura, Diseño y Estudios Urbanos; Pontificia Universidad Católica de Chile; ${ }^{2}$ Centro de Desarrollo Urbano Sustentable (CEDEUS); ${ }^{3}$ Escuela de Construcción; Universidad de las Américas; 4 Observatorio de Ciudades (OCUC); Facultad de Arquitectura, Diseño y Estudios Urbanos; Pontificia Universidad Católica de Chile; ${ }^{5}$ Instituto de Estudio Urbanos y Territoriales; Facultad de Arquitectura, Diseño y Estudios Urbanos; Pontificia Universidad Católica de Chile; ${ }^{6}$ instituto de Geografía; Facultad de Historia, Geografía y Ciencia Política; Pontificia Universidad Católica de Chile. * Correo de contacto: felipe.encinas@uc.cl
} 


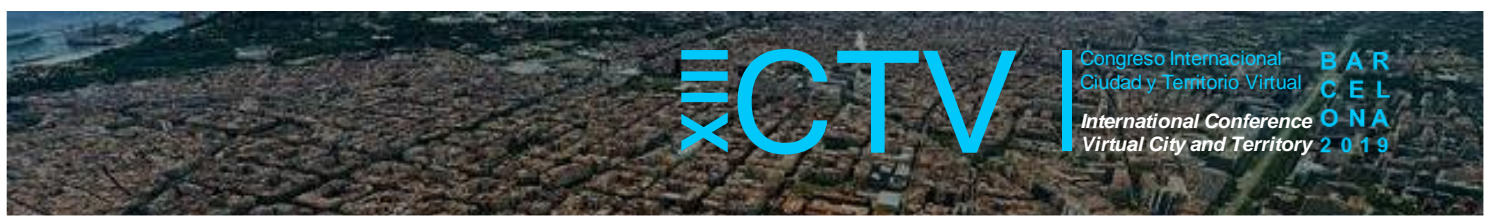

In this context, the incorporation of socio-spatial segregation methodologies to enrich this concept is of very recent date and has not yet been applied at the national level. This approach is important, since it allows identifying areas of agglomeration of energy poverty, an important aspect for the design of urban policies.

Palabras Clave: pobreza energética; segregación territorial; eficiencia energética

Key words: energy poverty; territorial segregation; energy efficiency

\section{Antecedentes}

El concepto de pobreza energética nace a principios de los años 90 en el Reino Unido como la incapacidad de obtener en un hogar la cantidad adecuada de servicios energéticos por el 10\% del ingreso familiar (Boardman 1991). Esta definición posteriormente ha sido complementada con aspectos constructivos y de habitabilidad, a partir de, por ejemplo, el establecimiento de una temperatura límite para la obtención de confort (DOE 1996). En la literatura internacional más reciente es posible encontrar definiciones con un fuerte sesgo interdisciplinar a partir de la accesibilidad a 3 umbrales de vulnerabilidad: tecnológico, físico y económico (González-Eguino 2015). Este último es el más ampliamente utilizado en países desarrollados, principalmente asociado al consumo de calefacción para uso doméstico (concepto más preciso de "fuel poverty"), aunque se reconoce que tiende a subestimar la cantidad de hogares que efectivamente no puede costear el consumo energético para estas mínimas condiciones de habitabilidad, o la provisión de otras demandas de energía, que constituyen el denominado umbral tecnológico (Mould and Baker 2017; Bouzarovski and Petrova 2015).

Por otra parte, el umbral físico ha sido estudiado de manera más reciente desde el confort térmico, específicamente en su relación con la vulnerabilidad y salud, encontrando correlaciones significativas con respecto a las características constructivas de las viviendas (Grey, Jiang, and Poortinga 2015; Atsalis et al. 2016). En este contexto, la incorporación de metodologías de segregación socio-espacial para enriquecer este concepto es de muy reciente data y todavía no ha sido aplicado a nivel nacional. Este enfoque resulta importante, puesto que permite identificar zonas de aglomeración de pobreza energética, aspecto importante para el diseño de políticas urbanas.

\section{Metodología}

Este trabajo propone una aproximación multidimensional con alcances socio-espaciales para abordar la temática de pobreza energética a nivel metropolitano de Santiago de Chile. Si consideramos la regla del $10 \%$ del ingreso familiar como punto de partida para formular una definición a nivel nacional, en teoría sería posible suponer que podría alcanzarse una determinada temperatura de confort. Sin embargo, la experiencia nacional -caracterizada en general por una baja calidad de envolvente térmica y sistemas ineficientes de ventilación y calefacción (Bustamante 2013; Celis et al. 2012; Caldera Sánchez 2012)- sugiere que difícilmente se pueda conseguir ese nivel de habitabilidad de manera permanente en la población más vulnerable, a un nivel de gasto que pudiese ser abordable. La pobreza energética establece entonces, una paradoja: la baja calidad habitacional no siempre conllevará un mayor consumo energético, puesto que muchas veces se estará tan lejos de la posibilidad de acceder a condiciones de habitabilidad adecuadas, que el gasto se reducirá al mínimo. Esta situación nos obliga a entender este fenómeno como la interacción de tres brechas: la de calidad constructiva-energética, la socioeconómica y la espacial-territorial. En ese sentido, se espera probar que, en un entorno de liberalización del mercado de viviendas, la 


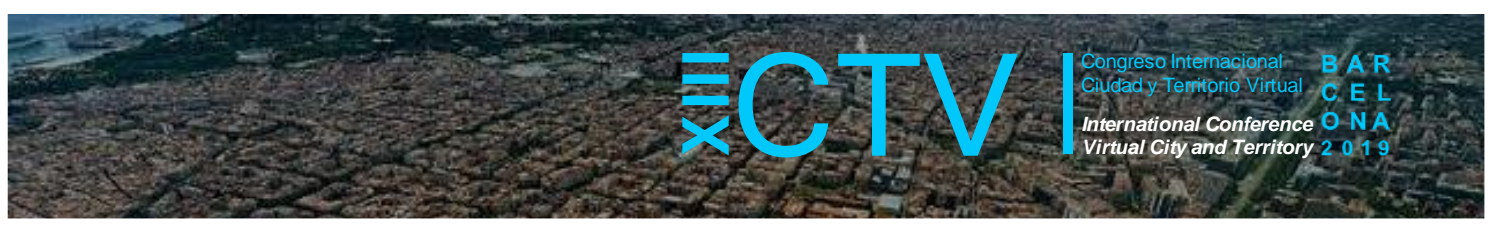

pobreza energética se encuentra aglomerada como consecuencia de la segregación socioespacial del ingreso.

Para relevar este fenómeno se realizó una aproximación basada en estadística espacial para el Área Metropolitana de Santiago (AMS), considerando datos de temperatura interior de las casas en invierno (recolectadas de la Red Nacional de Monitoreo, RENAM) y contrastándolas con variables territoriales atingentes, como la vitalidad de la biomasa espacialmente distribuida, temperatura superficial terrestre, amplitud térmica anual, materialidad de la vivienda y valores promedio del Indicador Socio Material Territorial (ISMT), proxy de ingreso, construido por el Observatorio de Ciudades UC.

Asumiendo una importante componente de autocorrelación espacial, dadas las lógicas de aglomeración socioeconómica, se llevaron a cabo análisis de clusterización local (Anselin 1995) y de regresiones ponderadas geográficamente (Fotheringham, Brundson and Charlton 2002) para dilucidar las lógicas de aglomeración de las variables y para medir la relación entre éstas, respectivamente. Cabe destacar que todas las regresiones testeadas fueron previamente abordadas a través de un ESDA (Análisis estadístico exploratorio) de forma tal de asegurar la consistencia de resultados, paro lo cual se llevaron a cabo test de pertinencia de las variables, multicolinealidad, análisis de condiciones y de clusterización de los residuos.

\section{Resultados}

De lo anterior se establecen tres resultados relevantes:

1. Existe una fuerte correlación entre las temperaturas interiores de las viviendas en invierno y las temperaturas externas (Coeficiente de determinación ajustado $=0.75$ ). El resultado de la regresión es mucho más potente si se segmenta espacialmente el análisis a partir de los grupos socioeconómicos, lo que da cuenta de un fenómeno particular. En muchas zonas (en amarillo, Figura 1) existe una relación directa entre la temperatura del exterior (del suelo) y de la casa, obviando todos los mitigantes como la aislación de la vivienda o la calefacción, lo que se explicaría por el nulo efecto de estas.

La zona en donde se subestima la predicción es en zonas de edificio de Santiago, Ñuñoa y Providencia, parte de Vitacura y paradójicamente algunas zonas periféricas como Puente Alto, esto indicaría que en dichas zonas (en rojo) las temperaturas son más altas que lo predicho por el modelo, por lo tanto, hay un efecto positio en las temperaturas interiores que podría explicarse por calefacción o aislamiento de las viviendas. En azul el patrón es inverso, es decir las temperaturas internas fueron sobrestimadas dado un comportamiento más cálido de las temperaturas exteriores; muchas de estas zonas corresponden a zonas del piedemonte, zonas con grandes parques (San Cristobal, Quinta Normal, Parque Intercomunal). Ciertas zonas pericentrales (Lo Espejo, La Cisterna o Maipú) no tienen una explicación clara, pero dado el agrupamiento se infiere un patrón de autocorrelación relevante. 


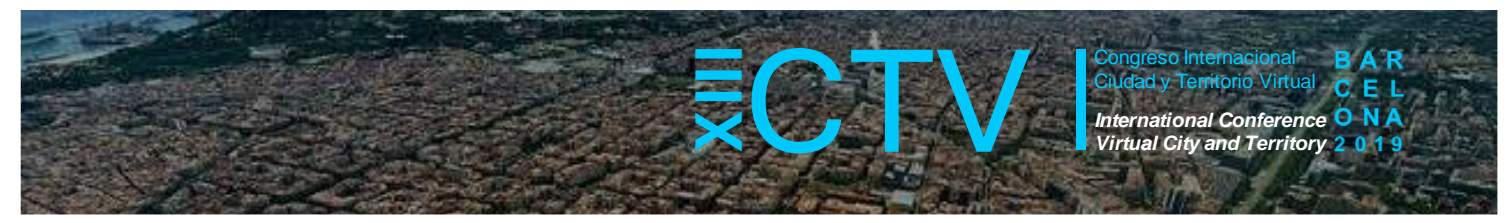

Figura 1. GWR TEMP LST invierno

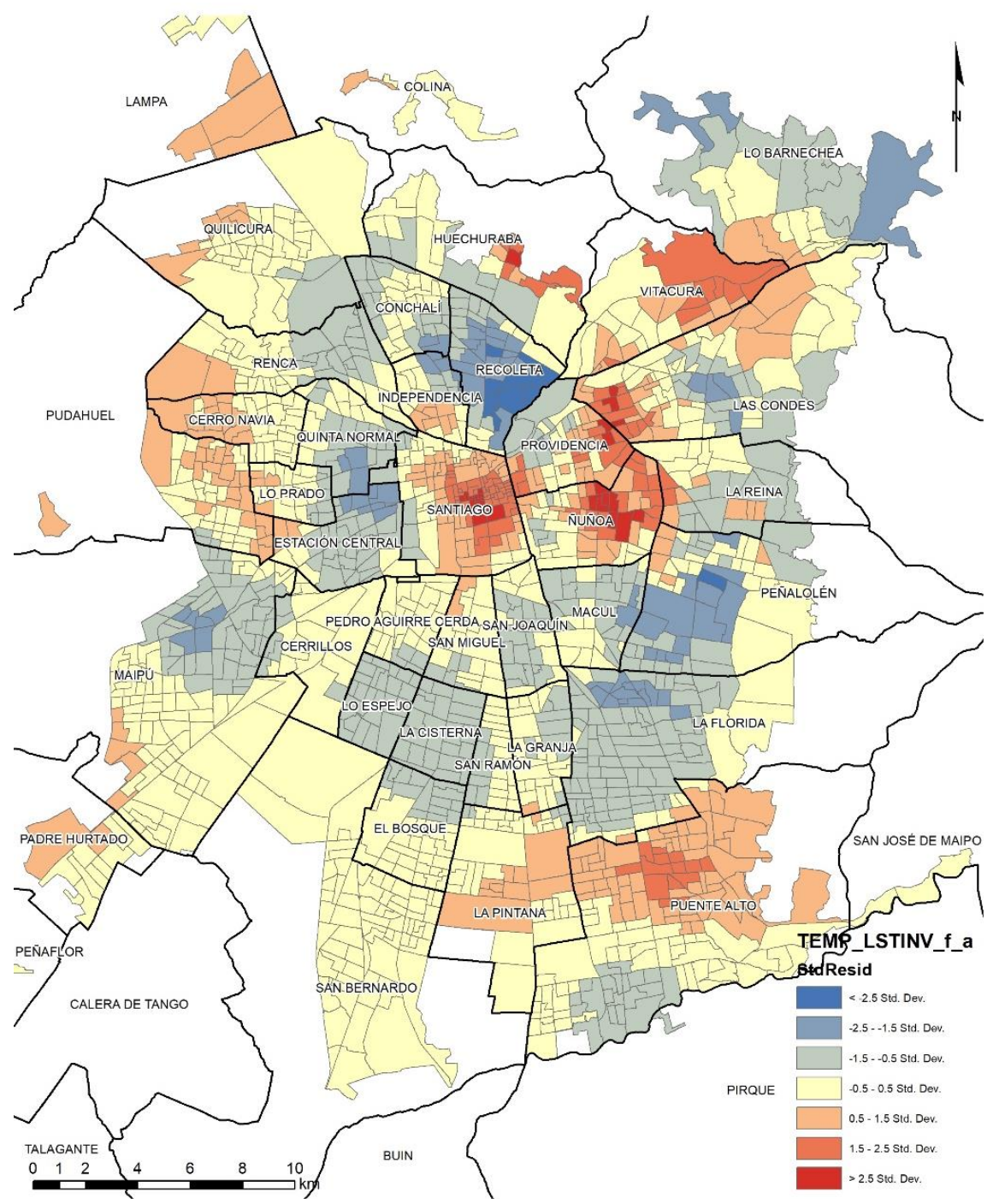

Nota: GWR: Temperatura interior LST invierno $(R 2: 0,74)$

Fuente: Elaboración propia.

2. Existe una relación importante, pero moderada, de la temperatura interior con la segmentación socioeconómica (coeficiente de correlación ajustado=0,47). La desviación estándar de los residuos da cuenta de una buena cantidad de zonas con ajuste adecuado. El cono de altos ingresos y el centro de Santiago muestran una subestimación de las temperaturas (ver, lo que da cuenta de manera teórica la importancia en estas zonas del uso de calefacción, probablemente relacionado también con la materialidad de la vivienda (también relacionado con los departamentos en zonas de alta densidad).

Al igual que en el caso anterior destacan zonas subestimadas (color azul) patrones probablemente explicados por la presencia de grandes paños de áreas verdes (parques urbanos) con un alto porcentaje de biomasa asociado, pero también con continuidad espacial (a diferencia de los casos asociados por ejemplo a biomasa en espacios privados, tales como patios de viviendas) 


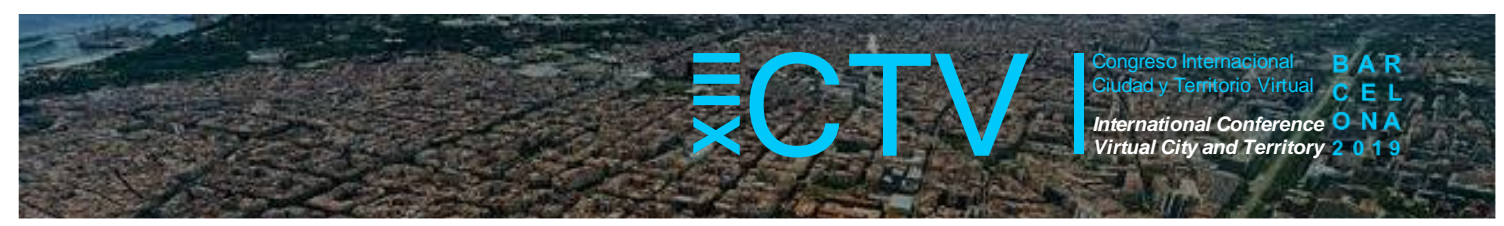

Figura 2. GWR TEMP ISMT

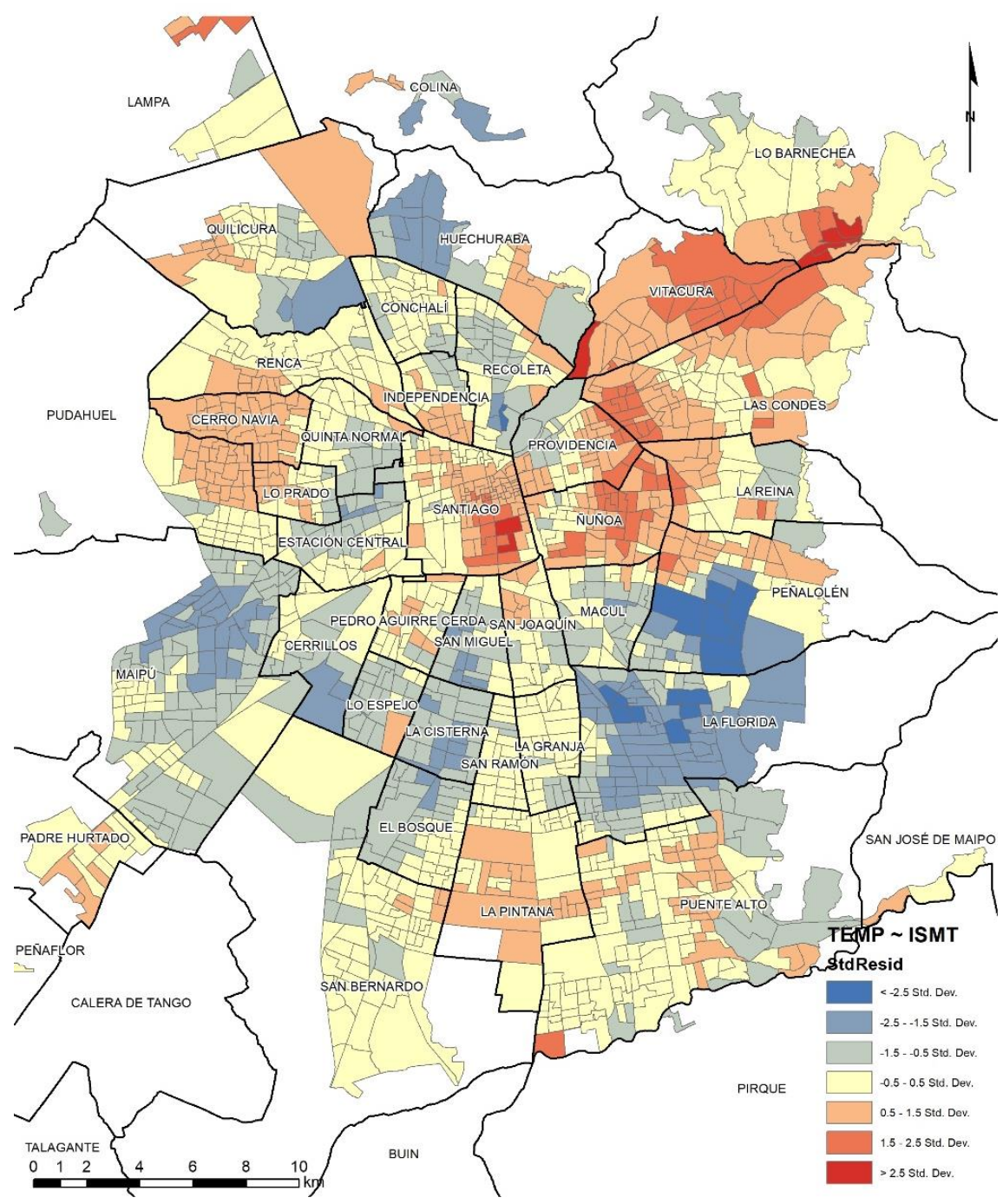

Nota: GWR: Temperatura interior ISMT (R2: 0,47)

Fuente: Elaboración propia.

3. Si bien la primera regresión entrega una mayor porcentaje de varianza explicado, podemos dilucidar ciertos patrones espaciales claros, vinculados al ingreso, la presencia de vegetación y la materialidad de la vivienda; esto permite establecer dos puntos importantes: a) la temperatura interior, como variable dependiente posee un grado de autocorrelación espacial relevante, justificado espacialmente por la correlación con variables conocidas por sus procesos de aglomeración espacial (como el ingreso y sus variables proxy); b) que, a modo de hipótesis, una regresión geográficamente ponderada con más variables independientes debiera tener un comportamiento adecuado, a menos que se produjera un alto grado de multicolinealidad o bien una aglomeración de residuos muy alta.

A modo de consideraciones metodológicas se puede agregar, además: 


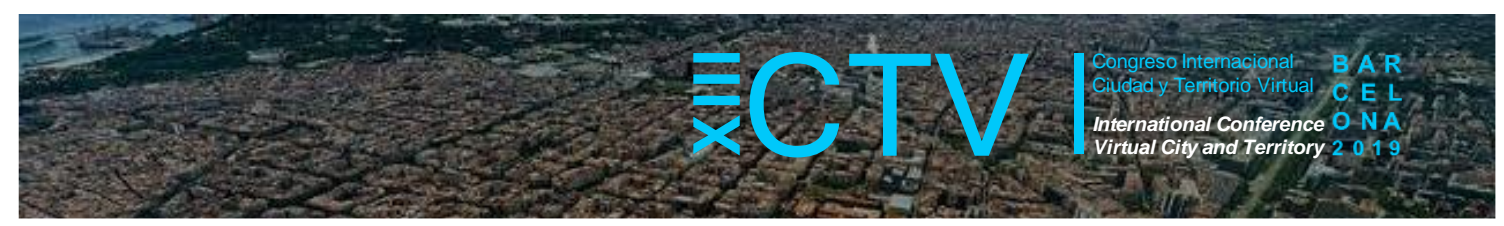

- Ningún modelo múltiple soporto el ISMT, generando éste problemas de diseño que invalidaron los resultados, esto fundamentado por su construcción multivariable lo que predispone a un diseño con multicolinealidad.

- El modelo más efectivo en ganancia de varianza explicada versus cantidad de variables fue la combinatoria entre NDVI (índice de vegetación normalizado) de Inverno con LST invierno (temperatura superficial terrestre), no obstante, se sabe que LST requiere de NDVI como variable por lo tanto se asumen procesos de multicolinealidad teórico, paradójicamente no relevados en la práctica $\left(R^{2}=0,59\right)$

- El aporte de una tercera variable, como la materialidad de la vivienda bajaba el coeficiente de determinación a $R^{2}=0,51$, por lo que no se consideró una adición efectiva.

- Se probará la adición de nuevas variables como parte del trabajo en progreso de la presente investigación, entre estás la vegetación de áreas verdes públicas en extensión (grandes parques urbanos)

Estos análisis permiten establecer que existe una clara autocorrelación espacial del entorno construido, medido en la capacidad de las envolventes térmicas de las viviendas en permitir o asegurar un rango de temperaturas, aglomerándose por estrato socioeconómico.

Esta evidencia resulta de particular importancia para profundizar el concepto de pobreza energética y discutir sobre políticas públicas en esa línea, particularmente por el hecho que ésta es en gran medida consecuencia de una segregación socioeconómica propia de ciudades con mercados liberalizados de vivienda (relación previamente estudiada en términos teóricos en Encinas, Aguirre, and Truffello 2019). Esto incluye no solamente los aspectos constructivos de la envolvente térmica, sino también los elementos asociados a la biomasa existente en el entorno inmediato, y las características socioeconómicas de los hogares.

Conflicto de Intereses: Los autores declaran que no hay conflicto de intereses.

\section{Referencias}

Anselin, L. (1995). "Local Indicators of Spatial Association-LISA". Geographical Analysis 27(2): 93-115. https://doi.org/10.1111/i.1538-4632.1995.tb00338.x

Atsalis, A., S. Mirasgedis, C. Tourkolias, and D. Diakoulaki. (2016). "Fuel Poverty in Greece: Quantitative Analysis and Implications for Policy." Energy and Buildings 131: 87-98. https://doi.org/10.1016/i.enbuild.2016.09.025

Boardman, B. (1991). Fuel Poverty: From Cold Homes to Affordable Warmth. London: Wiley and Sons.

Bouzarovski, Stefan, and Saska Petrova. (2015). "A Global Perspective on Domestic Energy Deprivation: Overcoming the Energy Poverty-Fuel Poverty Binary." Energy Research \& Social Science 10: 31-40.

Bustamante, Waldo. (2013). Informe Nº6. Evaluación Económica Social, Artículo 4.1.10 de La Ordenanza General de Urbanismo y Construcciones. Ministerio. Santiago. 


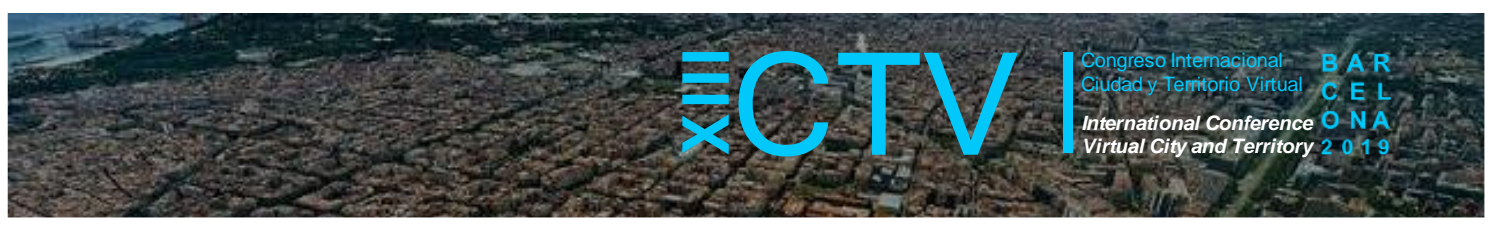

Caldera Sánchez, Aida. (2012). "Building Blocks for a Better Functioning Housing Market in Chile." Paris: OECD Publishing. https://doi.org/10.1787/5k9fj3hgsnvh-en

Celis, Flavio, Rodrigo García, Maureen Trebilcock, Olavo Escorcia, Underléa Miotto, and Muriel Diaz. (2012). "Análisis Energético de Las Viviendas Del Centro-Sur de Chile." Arquiteturarevista 8 (1): 62-75. https://doi.org/10.4013/arq.2012.81.07

DOE. (1996). English House Condition Survey 1991: Energy Report. London: Department of Environment.

Encinas, Felipe, Aguirre, Carlos, and Truffello, Ricardo. (2019). Raíces de la desigualdad: Impacto de la conformación del precio inmobiliario en la segregación urbana. In: "Políticas Públicas para la Equidad Social. Tomo II". Barcelona and Santiago: Universidad de Barcelona and Universidad de Santiago de Chile. Editores/as: Rivera-Vargas, Pablo, Muñoz-Saavedra, Judith, Morales-Olivares, Rommy, Butendieck, Stefanie.

González-Eguino, Mikel. (2015). "Energy Poverty: An Overview." Renewable and Sustainable Energy Reviews 47: 377-85. https://doi.org/10.1016/j.rser.2015.03.013

Fotheringham, A., Brunsdon, C., \& Charlton, M. (2002). Geographically weighted regression: the analysis of spatially varying relationships. New Jersey: John Wiley \& Sons, Inc.

Grey, Charlotte, Shiyu Jiang, and Wouter Poortinga. 2015. "Fuel Poverty, Thermal Comfort, and Health in Low-Income Areas in Wales: Results from the First Wave of Data Collection for the Arbed Health Impact Study." Recuperado de http://www.cardiff.ac.uk/architecture/files/2015/03/WSA-Working-Paper-02-2015.pdf

Mould, Ronald, and Keith J. Baker. 2017. "Documenting Fuel Poverty from the Householders' Perspective." Energy Research and Social Science 31: 21-31. https://doi.org/10.1016/i.erss.2017.06.004 